Proceedings

\title{
Sub-100 nm Chitosan-Triphosphate-DNA Nanoparticles for Delivery of DNA Vaccines ${ }^{+}$
}

\author{
Renato Nunes 1,2,*, Ângela Sousa ${ }^{1}$, Aiva Simaite ${ }^{2}$, Ahmed Aido ${ }^{2}$ and Matej Buzgo ${ }^{2}$ \\ 1 CICS-UBI - Health Sciences Research Center, University of Beira Interior, Avenida Infante D Henrique, \\ 6200-506 Covilhã, Portugal; angela@fcsaude.ubi.pt \\ 2 InoCure s.r.o, R\&D Lab, Prumyslová 1960, 25088 Celákovice, Czech Republic; aiva@inocure.cz (A.S.); \\ ahmed@inocure.cz (A.A.); matej@inocure.cz (M.B.) \\ * Correspondence: renato.nunes@ubi.pt; Tel.: +420-607-722-205 \\ + Presented at the 1st International Electronic Conference on Pharmaceutics, 1-15 December 2020; \\ Available online: https://iecp2020.sciforum.net/.
}

Citation: Nunes, R.; Sousa, Â.; Simaite, A.; Aido, A.; Buzgo, M. Sub100 nm Chitosan-TriphosphateDNA Nanoparticles for Delivery of DNA Vaccines. Proceedings 2021, 78, 12. https://doi.org/10.3390/IECP202008653

Published: 1 December 2020

Publisher's Note: MDPI stays neutral with regard to jurisdictional claims in published maps and institutional affiliations.

Copyright: $\left(C^{2} 221\right.$ by the authors. Licensee MDPI, Basel, Switzerland. This article is an open access article distributed under the terms and conditions of the Creative Commons Attribution (CC BY) license (http://creativecommons.org/licenses/by/4.0/).

\begin{abstract}
Intramuscular delivery is one of the main routes for DNA vaccines administration. However, it requires large amounts of the DNA to be administered and external stimulation to encourage the internalization of the DNA. In this work, we consider alternative routes for less invasive administration and develop drug delivery systems (DDS) for intranasal administration. Chitosan polyplexes using sodium tripolyphosphate (TPP) as a crosslinker were prepared using the ionic gelation method. Our method allowed preparation of nanoparticles with the size below $50 \mathrm{~nm}$, which is at least two times lower than previously reported sizes. Moreover, despite the small sizes, we obtained DNA encapsulation efficiencies of about $70 \%$. Parameters that may affect the encapsulation efficiency were investigated, including different TPP-chitosan ratios and concentrations of DNA. We found that encapsulation efficiency of DNA inside the particles decreases with the increasing TPP-chitosan ratio. Moreover, increasing the DNA concentration leads to a higher encapsulation efficiency. Small $(<50 \mathrm{~nm})$ chitosan nanoparticles hold enormous potential as DNA carriers due to their physiological barriers and subsequent internalization.
\end{abstract}

Keywords: chitosan; DNA vaccines; tripolyphosphate; nanoparticles; ionic gelation

\section{Introduction}

Cancer is the second most prevalent cause of death in the world that does not have a universal cure. Human papilloma virus (HPV) is among the main carcinogenic pathogens and its infection is related to several cancers, such as cervical and oropharyngeal [1,2]. Cervical cancer is the 4th largest cause of cancer in women worldwide [3]. The prophylactic vaccination against the human papilloma virus (HPV) infection that is already commercially available, Gardasil ${ }^{\circledR}$, from Merck, prevents infection from HPV-16 and 18, which are responsible for around $70 \%$ of cervical cancers [4]. However, the current vaccine does not have a therapeutic effect against the already infected cells. That is, the vaccine can prevent the infection by HPV, but cannot prevent the development of cancers from pre-existing infections [5]. Therapeutic cancer vaccines are still a major area of ongoing research.

One of the promising approaches for therapeutic vaccines is DNA vaccination. DNA vaccination is applicable against a great range of viral, bacterial, and parasitic diseases, including HPV [6,7]. In comparison with traditional vaccines, DNA vaccines have numerous promising advantages. They are simpler to synthesize and can be produced on a large scale. In general, they are considered to be safer, since the pathogen is not required in the vaccine production. Additionally, DNA vaccines do not require refrigeration for storage, transport, and distribution, which may allow for easier distribution worldwide $[8,9]$. DNA vaccines are particularly appropriate for antitumor and anticancer treatment 
due to the fact that their encoded antigen can be expressed inside the antigen presenting cells (APCs). These cells can then activate the needed immune response for the dissolution or destruction of a recognized infected cell $[10,11]$. However, the delivery of the DNA vaccines to the APCs remains challenging. In this work, we evaluate ionotropic chitosan gelation as a potential method for the encapsulation and delivery of DNA vaccines.

Successful drug delivery systems (DDS) should provide a number of features, such as the ability to penetrate through several anatomical barriers, sustained and controlled release of their active pharmaceutical ingredients (APIs) as well as locally, stable and deep tissue penetration. These features can be enabled by nanometer-sized carriers made of synthetic and natural polymers. Nanocarriers can also be used for drug delivery through mucosal layers, and help the cellular internalization of the API [12,13]. Ionotropic gelation is one of the most common methods used for the encapsulation and delivery of DNA. The nanoparticles are produced by mixing the ionic crosslinker, tripolyphosphate (TPP), with the cationic polymer, chitosan (CS), under constant magnetic stirring. Chitosan is known as a good carrier for the delivery and internalization of non-viral vectors for gene delivery. The polycationic nature of CS enables electrostatic complexation with the DNA, which under controlled conditions would lead to the formation of DNA-CS complexes. With the optimum concentrations and other conditions, nanoparticles with sizes below $100 \mathrm{~nm}$ can be formed [14-17]. However, such small particles are rarely reported.

In this work, we developed and optimized the ionotropic gelation method for the encapsulation of DNA in order to produce nanoparticles with sizes ranging from 30 to $60 \mathrm{~nm}$. We demonstrated that the method is robust and reproducible and, moreover, leads to high DNA encapsulation efficiency.

\section{Materials and Methods}

\subsection{Materials}

Medical grade chitosan 95/1000 was purchased from Heppe Medical, sodium tripolyphosphate (TPP) was obtained from Across Organics, deoxyribonucleic acid sodium salt (DNA) was acquired from $\mathrm{MPBIO}, 35 \%$ hydrochloric acid $(\mathrm{HCl})$, while sodium hydroxide palettes $(\mathrm{NaOH})$ and glacial acetic acid (AA) were all acquired from VWR. The following solutions were freshly prepared by using deionized water from VWR: $2 \mathrm{M} \mathrm{HCl}, 10 \mathrm{M} \mathrm{NaOH}, 1 \%$ and $2 \%(v / v)$ acetic acid.

\subsection{Methods}

\subsubsection{Ionotropic Gelation}

Ionotropic gelation using chitosan as a polymer and TPP as a crosslinker was used to prepare the nanoparticles. Particles with and without DNA were prepared using the same procedure adopted from [18] with slight modifications. Particles without DNA were used as a negative control. The process of ionic gelation is depicted in Figure 1. Next, $0.1 \%(w / v)$ chitosan (CS) in $1 \%(v / v)$ acetic acid and $0.1 \%(w / v)$ of TPP solution in water were prepared as stock solutions. The $\mathrm{pH}$ level of the chitosan and TPP solutions were adjusted to 5.2-5.5 and $\mathrm{pH} 2$ by the addition of $\mathrm{NaOH}$ and $\mathrm{HCl}$, respectively. For the experiments with DNA encapsulation, the DNA was dissolved in the TPP solution to reach the needed concentration. Then, both chitosan and TPP solutions were filtered using a $0.45 \mu \mathrm{m}$ polyethersulfone syringe filter (from VWR). A syringe pump (New Era Pump System, Inc., Farmingdale, NY, USA) was used to add TPP solution to the chitosan solution dropwise with the flow rate of $0.25 \mathrm{~mL} / \mathrm{min}$. To ensure there was a consistent drop size, needle size 20 was used in all experiments, leading to an addition rate of about 15-16 drops/min. During the addition, the chitosan solution was stirred vigorously (600 rpm) using a magnetic stirrer. The solution was mixed for an additional $30 \mathrm{~min}$ after all of the TPP was consumed. All experiments were done at room temperature. As a positive control, $0.1 \%(w / v)$ deoxyribonucleic acid sodium salt (DNA) in water was prepared and mixed with the blank particles without DNA in a 1:1 ratio (by volume). A combined 
solution was incubated for $2 \mathrm{~h}$ at room temperature for DNA adsorption to chitosan-TPP nanoparticles to take place.

All experiments were performed in triplicates and the results are presented as means \pm standard deviation (SD).

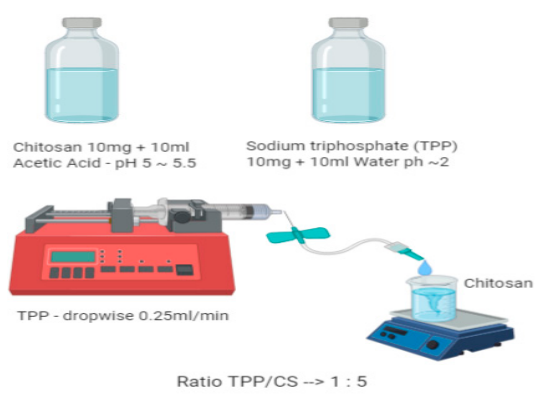

(a)

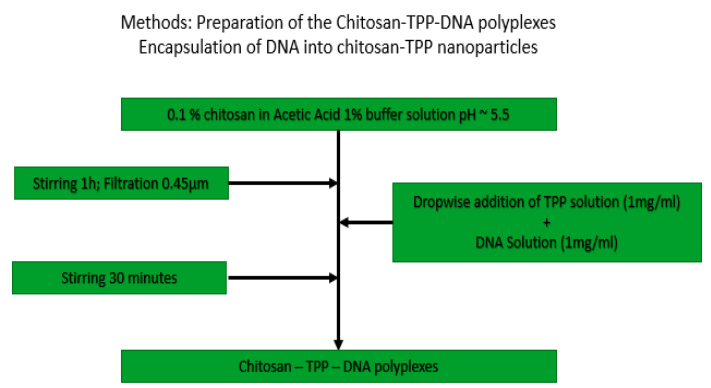

(b)

Figure 1. (a) Schematic illustration of chitosan-tripolyphosphate (TPP) nanoparticles (b) Flow chart of the nanoparticle with DNA preparation. First, solutions with optimized concentrations were prepared. Then, TPP with DNA was added to the chitosan solution dropwise while stirring.

\subsubsection{Encapsulation Efficiency}

To estimate the amount of the DNA encapsulated in the chitosan NPs, the NPs solution was spun down using Centurion Scientific Benchtop Centrifuge for $20 \mathrm{~min}$ at 22.000 $\mathrm{RCF}$ and the DNA concentration in the supernatant was measured. The indirect encapsulation efficiency (iEE) was calculated as in (1), where c(total) is the theoretical DNA concentration in the solution and c(sup) is the measured DNA concentration after the encapsulation.

$$
i E . E . \%=\frac{c(\text { total })-\mathrm{c}(\sup )}{c(\text { total })} \times 100 \%
$$

To verify the accuracy of the method, the amount of the encapsulated DNA was also estimated in the particles (dEE). NPs with and without DNA were spun down for $20 \mathrm{~min}$ at $22.000 \mathrm{RCF}$. The supernatant was removed for iEE measurement and the equivalent volume $(1 \mathrm{~mL})$ of $2 \%$ acetic acid and an additional $100 \mu \mathrm{L}$ of $2 \mathrm{M} \mathrm{HCl}$ were added. The solution was vortexed for one minute and then sonicated using the ultrasound homogenizer (from Qsonica sonicators) for $30 \mathrm{~s}$ at $40 \%$ amplitude. The solution was spun down for $20 \mathrm{~min}$ at $22.000 \mathrm{RCF}$ mode to make sure that no pellet was formed and the DNA concentration was measured. The dEE was calculated as a ratio of measured and theoretical DNA concentrations.

In both cases, DNA concentration was used spectrophotometrically. That is, supernatant or dissolved nanoparticle solution $(1 \mathrm{~mL})$ was added to the quartz cuvette. The solution was diluted 2 to 3 times with $1 \%$ acetic acid, and the absorbance was measured using a cuvette reader from SpectraMax. The absorbance value at $260 \mathrm{~nm}$ was recorded.

\subsubsection{Particle Size Determination}

The size of the prepared CS-TPP-DNA-polyplexes was also analyzed using the Nanophox Dynamic Light Scattering (DLS) with photon cross-correlation spectroscopy from Sympatec. The particles were analyzed immediately after the preparation and after $72 \mathrm{~h}$ to evaluate the stability of the particle suspension. All DLS experiments were carried out at a temperature of $25^{\circ} \mathrm{C}$. 


\section{Results}

Ionotropic gelation is a popular method for DNA encapsulation. Multiple authors have demonstrated the ability to prepared nanoparticles with DNA that are below $300 \mathrm{~nm}$ [18-20]. Liping et al. has shown that particles smaller than $200 \mathrm{~nm}$ can be prepared by using a solution with low chitosan and TPP concentrations of $0.1 \%$ [19]. However, to the best of our knowledge, nanoparticles with a size below $100 \mathrm{~nm}$ have not yet been prepared. In this work, we have used low solution concentrations and slow controlled drop-wise addition in order to make nanoparticles with a size of around $30-60 \mathrm{~nm}$. We investigated the influence of the TPP/CS ratio and DNA concentration on the size and EE of formed nanoparticles.

\subsection{Influence of Changing the TPP/CS Ratio on NPs Size and dEE}

To investigate the influence of the TPP/CS ratio on nanoparticle size and DNA encapsulation efficiency, four NPs solutions with TPP/CS ratios of 1:5, 1.25:5, 1.5:5, and 2:5 were prepared. In these experiments, $35 \mu \mathrm{g} / \mathrm{mL}$ DNA was used. The formulation of prepared samples as well as their physicochemical characteristics are summarized in Table 1. As shown in Figure 2a, using the ionotropic gelation with controlled addition speed, very small nanoparticles of 30-50 nm were obtained. Only the particles with a high TPP/CS ratio led to the formation of larger particles of about $57 \mathrm{~nm}$. In all cases, the particle size did not change significantly after storing them in the fridge for $72 \mathrm{~h}$. Only a slight increase of about 2-6 nm was detected. However, as shown in Table 1, the formed particles were not monodispersed and had polydispersity index results of $>0.5$.

Table 1. Average particle size, Polydispersity Index (PDI), and encapsulation efficiency of chitosan-TPP-DNA polyplexes with different TPP/CS ratios.

\begin{tabular}{cccccc}
\hline TPP/CS Ratio & $\begin{array}{c}\text { Z-Average Size } \\
(\mathbf{n m})\end{array}$ & Polydispersity Index (PDI) & $\begin{array}{c}\text { Z-Average Size after } \\
\mathbf{7 2} \mathbf{~ h}(\mathbf{n m})\end{array}$ & $\begin{array}{c}\text { (PDI) after } \\
\mathbf{7 2} \mathbf{~ h}\end{array}$ & $\begin{array}{c}\text { Encapsulation } \\
\text { Efficiency (\%) }\end{array}$ \\
\hline $1: 5$ & $45 \pm 0.5$ & $0.55 \pm 0.01$ & $47 \pm 6.9$ & $0.51 \pm 0.006$ & $77 \pm 10$ \\
$1.25: 5$ & $37 \pm 0.3$ & $0.50 \pm 0.03$ & $40 \pm 0.9$ & $0.48 \pm 0.004$ & $73 \pm 5$ \\
$1.5: 5$ & $39 \pm 1.7$ & $0.46 \pm 0.04$ & $45 \pm 0.9$ & $0.40 \pm 0.009$ & $69 \pm 4$ \\
$2: 5$ & $57 \pm 0.8$ & $0.50 \pm 0.02$ & $63 \pm 1.9$ & $0.46 \pm 0.010$ & $66 \pm 4$ \\
\hline
\end{tabular}

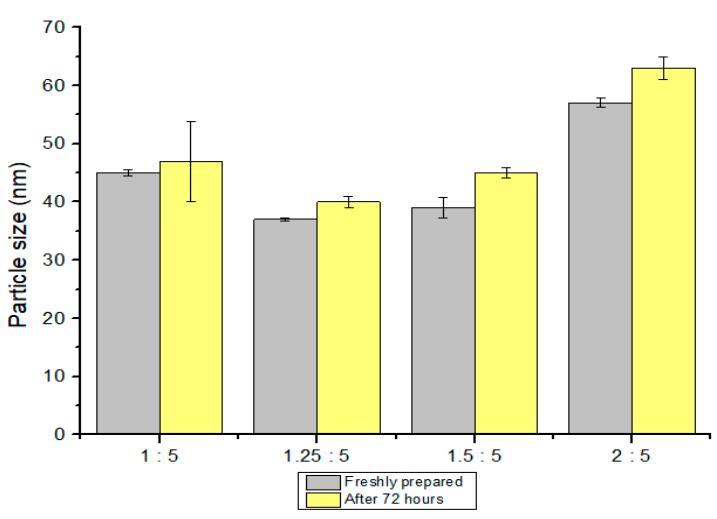

(a)

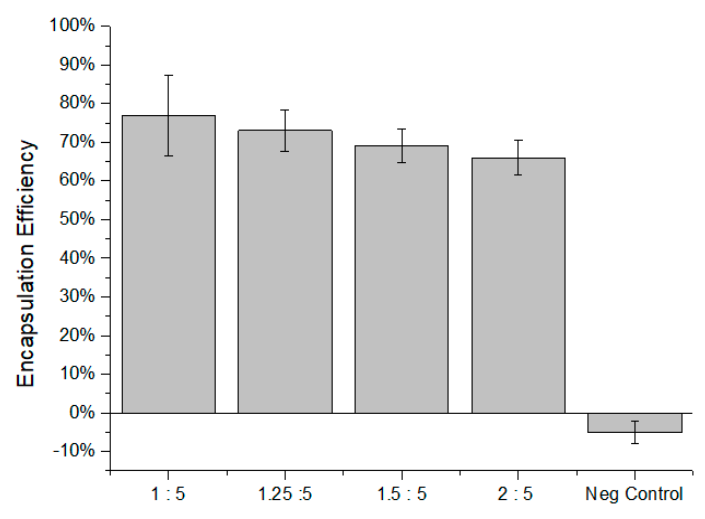

(b)

Figure 2. (a) Particle size (nm) freshly prepared and after $72 \mathrm{~h}$ with different TPP/CS ratios; (b) Results of the encapsulation efficiency with different TPP/CS ratios.

The amount of the DNA encapsulated in the microparticles was measured using the direct method after the extraction of the DNA from the collected nanoparticles. Prior to the measurement, the amount of the DNA that was adsorbed on the surface of the NPs was evaluated by mixing the prepared blank NPs with the DNA solution and measuring 
the concentration of the DNA in the supernatant. A total of $35 \mu \mathrm{g} / \mathrm{mL}$ of DNA was added, and $-0.98 \pm 1.21 \mu \mathrm{g} / \mathrm{mL}$ was measured (not shown), indicating that only about $3 \%$ of the DNA may be adsorbed on the NP's surface. As shown in Figure $2 b$, the negative control showed that there is no interference between the excipients used in particle preparation. As shown in Figure $2 b$, all formulations showed a good efficiency of encapsulation with measured dEE of $77 \%, 73 \%, 69 \%$, and $66 \%$ for samples 1 to 4 , respectively. Increasing the $\mathrm{TPP} / \mathrm{CS}$ ratio led to a small decrease in the encapsulation efficiency but only of about $10 \%$.

\subsection{Influence of Changing the DNA Concentration in NP Size and $d E E$}

To investigate the influence of the DNA concentration on the nanoparticles size and DNA encapsulation efficiency, four formulations of NPs with DNA concentrations of 15, 25,35 , and $45 \mu \mathrm{g} / \mathrm{mL}$ were prepared. As mentioned before, particle size was measured immediately after the preparation and after $72 \mathrm{~h}$ to evaluate the NPs stability. The results are summarized in Table 2 and depicted in Figure 3 a,b. In all cases, very small nanoparticles, below $40 \mathrm{~nm}$ were formed. The particle size increased slightly after storage, yet the solution could be considered stable. A small increase of about $10 \mathrm{~nm}$ could be detected in the sample with $15 \mu \mathrm{g} / \mathrm{mL}$.

Table 2. Average particle size, PDI, and encapsulation efficiency of chitosan-TPP-DNA polyplexes with different DNA concentrations.

\begin{tabular}{cccccc}
\hline $\begin{array}{c}\text { DNA Concentra- } \\
\text { tion }(\boldsymbol{\mu g} / \mathbf{m L})\end{array}$ & $\begin{array}{c}\text { Z-Average Size } \\
(\mathbf{n m})\end{array}$ & $\begin{array}{c}\text { Polydispersity Index } \\
\mathbf{( P D I )}\end{array}$ & $\begin{array}{c}\text { Z-Average Size after 72 } \\
\mathbf{h}(\mathbf{n m})\end{array}$ & $\begin{array}{c}\text { (PDI) after } \\
\mathbf{7 2} \mathbf{~ h}\end{array}$ & $\begin{array}{c}\text { Encapsulation } \\
\text { Efficiency }(\mathbf{\%})\end{array}$ \\
\hline $15 \pm 5$ & $40 \pm 0.8$ & $0.59 \pm 0.01$ & $50 \pm 4.8$ & $0.39 \pm 0.26$ & $56 \pm 28$ \\
$25 \pm 5$ & $35 \pm 0.5$ & $0.48 \pm 0.05$ & $35 \pm 0.5$ & $0.53 \pm 0.006$ & $60 \pm 13$ \\
$35 \pm 5$ & $36 \pm 0.8$ & $0.47 \pm 0.05$ & $35 \pm 0.3$ & $0.56 \pm 0.006$ & $59 \pm 4$ \\
$45 \pm 5$ & $38 \pm 0.7$ & $0.47 \pm 0.04$ & $36 \pm 0.4$ & $0.55 \pm 0.01$ & $69 \pm 2$ \\
\hline
\end{tabular}

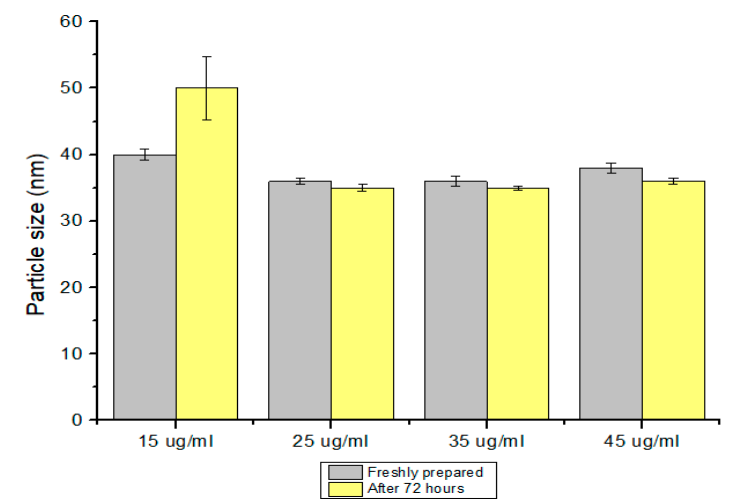

(a)

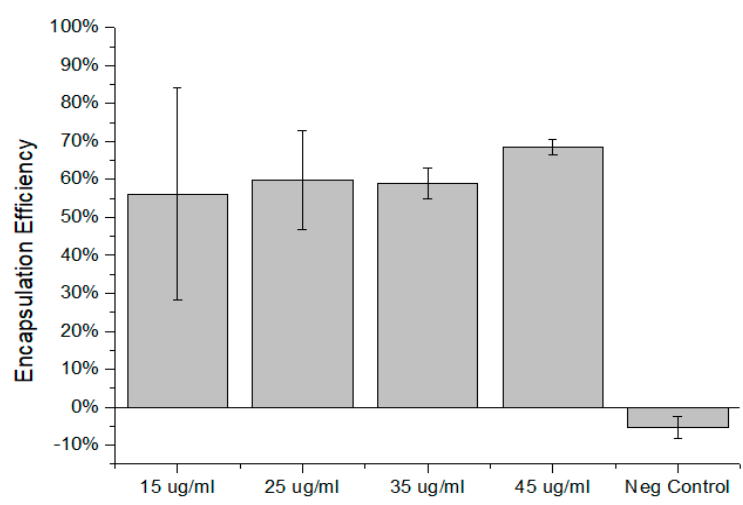

(b)

Figure 3. (a) Particle sizes (nm) freshly prepared and after $72 \mathrm{~h}$ with different DNA concentrations; (b) Results of the encapsulation efficiency with different DNA concentrations.

The encapsulation efficiency measurements of nanoparticles with different amounts of DNA added were evaluated as described before. As shown in Figure 3b, in all cases good encapsulation efficiencies were reached, with dEE results of $56 \%, 60 \%, 59 \%$, and $69 \%$ for samples 5 to 8 , respectively. Overall, our results suggest that higher encapsulation efficiency can be obtained with the increasing concentration of DNA. 


\section{Discussion and Future Work}

In this work, we found that increasing the TPP/CS ratio does not influence the particle size and encapsulation efficiency. This result had also been reported previously by Turan and Akbuğa [18]. Similar results were observed by Liping et al. [19]. Increasing the polymer concentration leads to an increase in particle size, while the influence of the polymer concentration was noted by Liping et al. [19]. The effect of chitosan molecular weight was studied by Huang et al. [20]. They have shown that the low molecular weight of chitosan may lead to nanoparticles that are smaller than $70 \mathrm{~nm}$. In this work, we used low molecular weighted medical purity chitosan, explored low solution concentrations, and obtained particles that are mostly below $50 \mathrm{~nm}$. The reasons behind such small particle sizes are still under investigation. We believe that one of the factors influencing the size is the polymer mixing rate, which is controlled by both the flow rate of the dropwise addition and the stirring rate. Indeed, our preliminary experiments suggest that a faster addition or larger droplet size may lead to larger nanoparticles. The influence of the addition rate on the ionotropic CS-DNA-TPP gelation has not yet been reported.

Compared to the other reported studies, we have measured slightly lower DNA encapsulation efficiencies - usually below $80 \%$, while Turan and Akbuğa reported around a $90 \%$ encapsulation efficiency [18]. This may be one of the drawbacks of extremely small nanoparticles. Our future work will focus on the optimization of the CS-DNA-TPP nanoparticles for the DNA internalization in eukaryotic cells. Depending on the successful results, low EE can be compensated for by the easier NP uptake caused by targeted cells. Moreover, the influence of the nanoparticle size on the mucopenetration and mucoadhesion will be tested to evaluate the feasibility of the intranasal administration.

\section{Conclusions}

Ionic gelation of CS/TPP nanoparticles is a promising delivery system for nucleic acids such as plasmid DNA. We have shown that these carriers can be downscaled below $50 \mathrm{~nm}$, which may enable more efficient internalization of such NPs by relevant cells. Moreover, we have shown that good quantities of DNA can be encapsulated using the CS/TPP nanoparticles. This is especially promising for the delivery of the DNA vaccines, as high quantities of the DNA are needed to achieve effective immunization and therapeutic effects. In further studies, we will evaluate the cellular uptake and the mucopenetration/mucoadhesion of these particles.

\section{Abbreviations}

The following abbreviations are used in this manuscript:

AA: Acetic acid; API: Active pharmaceutical ingredients; APC: Antigen presenting cells; CS: Chitosan; DDS: Drug delivery systems; dEE: Direct encapsulation efficiency; DLS: Dynamic Light Scattering; DNA: Deoxyribonucleic acid; EE: Encapsulation efficiency; $\mathrm{HCl}$ : Hydrochloric acid; HPV: Human papilloma virus; iEE: Indirect encapsulation efficiency; MHC: Major histocompatibility complex; MRT: Mean residence time; $\mathrm{NaOH}$ : Sodium hydroxide; NPs: Nanoparticles; pDNA: plasmid DNA; Rpm: Revolutions per minute; SD: Standard deviation; TPP: Sodium tripolyphosphate.

Author Contributions: R.N. designed the study, performed the experiments, conducted data processing, conducted analysis of the results and wrote the paper; Â.S. helped with designing the study, provided supporting material and reviewed the paper; A.S. guided the design of work and experiments, helped with the data analysis and writing of the manuscript; A.A. helped with method development, supervised the work and contributed with insights and discussions; M.B. helped with the development of the experiments, as well as with discussions and ideas. All authors have read and agreed to the published version of the manuscript.

Institutional Review Board Statement: Not applicable.

Informed Consent Statement: Not applicable. 
Data Availability Statement: The data presented in this study are available on request from the corresponding author.

Acknowledgments: R.N. has received support from the Erasmus+ traineeship program for his research activities at InoCure s.r.o. The project was supported by TACR project number FW01010445.

Conflicts of Interest: The authors declare no conflict of interest. The founding sponsors had no role in the design of the study; in the collection, analyses, or interpretation of data; in the writing of the manuscript, and in the decision to publish the results.

\section{References}

1. Nagai, H.; Kim, Y.H. Cancer prevention from the perspective of global cancer burden patterns. J. Thorac. Dis. 2017, 9, 448-451, doi:10.21037/jtd.2017.02.75.

2. Doorbar, J.; Egawa, N.; Griffin, H.; Kranjec, C.; Murakami, I. Human papillomavirus molecular biology and disease association. Rev. Med. Virol. 2015, 25, 2-23.

3. Arbyn, M.; Weiderpass, E.; Bruni, L.; de Sanjosé, S.; Saraiya, M.; Ferlay, J.; Bray, F. Estimates of incidence and mortality of cervical cancer in 2018: A worldwide analysis. Lancet Glob. Health 2020, 8, e191-e203, doi:10.1016/S2214-109X(19)30482-6. PMID: 31812369; PMCID: PMC7025157.

4. Padmanabhan, S.; Amin, T.; Sampat, B.; Cook-Deegan, R.; Chandrasekharan, S. Intellectual property, technology transfer and manufacture of low-cost HPV vaccines in India [published correction appears in Nat Biotechnol. 2012 Feb;30(2):193]. Nat. Biotechnol. 2010, 28, 671-678, doi:10.1038/nbt0710-671.

5. Almeida, A.M.; Queiroz, J.A.; Sousa, F.; Sousa, A. Cervical cancer and HPV-Infection: Ongoing therapeutic research to counteract the action of E6 and E7 oncoproteins. Drug Discov. Today 2019, 24, 2044-2057 doi:10.1016/j.drudis.2019.07.011.

6. Ha, S.; Jeon, B.; Youn, J.; Kim, S.; Cho, S.; Sung, Y. Protective effect of DNA vaccine during chemotherapy on reactivation and reinfection of Mycobacterium tuberculosis. Gene Ther. 2005, 12, 634-638.

7. Okuda, K.; Xin, K.Q.; Haruki, A.; Kawamoto, S.; Kojima, Y.; Hirahara, F.; Okada, H.; Klinman, D.; Hamajima, K. Transplacental genetic immunization after intravenous delivery of plasmid DNA to pregnant mice. J. Immunol. 2001, 167, 5478-5484.

8. Smith, H.A.; Klinman, D.M. The regulation of DNA vaccines. Curr. Opin. Biotechnol. 2001, 12, $299-303$.

9. Kutzler, M.A.; Weiner, D.B. DNAvaccines: Ready for prime time? Nat. Rev. Genet. 2008, 9, 776-788.

10. Anderson, R.J.; Schneider, J. Plasmid DNA and viral vector-based vaccines for the treatment of cancer. Vaccine 2007, 25 (Suppl. 2), B24-B34.

11. Lee, J.; Arun Kumar, S.; Jhan, Y.Y.; Bishop, C.J. Engineering DNA vaccines against infectious diseases. Acta Biomater. $2018,80,31$.

12. Mukherjee, S.; Ray, S.; Thakur, R.S. Solid lipid nanoparticles: A modern formulation approach in drug delivery system. Indian J. Pharm. Sci. 2009, 71, 349-358, doi:10.4103/0250-474x.57282.

13. Panyam, J.; Labhasetwar, V. Biodegradable nanoparticles for drug and gene delivery to cells and tissue. Adv. Drug Deliv. Rev. 2003, 55, 329-347.

14. López-León, T.; Carvalho, E.L.; Seijo, B.; Ortega-Vinuesa, J.L.; Bastos-González, D. Physicochemical characterization of chitosan nanoparticles: Electrokinetic and stability behavior. J. Colloid Interface Sci. 2005, 283, 344-351, doi:10.1016/j.jcis.2004.08.186. PMID: 15721903.

15. Morimoto, M.; Saimoto, H.; Usui, H.; Okamoto, Y.; Minami, S.; Shigemasa, Y. Biological activities of carbohydrate-branched chitosan derivatives. Biomacromolecules 2001, 2, 1133-1136.

16. Li, X.B.; Tsushima, Y.; Morimoto, M.; Saimoto, H.; Okamoto, Y.; Minami, S.; Shigemasa, Y. Biological activity of chitosan-sugar hybrids: Specific interaction with lectin. Polym. Adv. Technol. 2000, 11, 176-179.

17. Di Martino, A.; Sittinger, M.; Risbud, M.V. Chitosan: A versatile biopolymer for orthopaedic tissue-engineering. Biomaterials 2005, 26, 5983-5990.

18. Özbaş-Turan, S.; Akbuğa, J. Plasmid DNA-loaded chitosan/TPP nanoparticles for topical gene delivery. Drug Deliv. 2011, 18, 215-222, doi:10.3109/10717544.2010.544688. PMID: 21226549.

19. Wang, L.; Zhang, W.; Zhou, Q.; et al. Establishing Gene Delivery Systems Based on Small-Sized Chitosan Nanoparticles. J. Ocean Univ. China 2018, 17, 1253-1260, doi:10.1007/s11802-018-3658-8.

20. Huang, K; Sheu, Y; Chao, I. Preparation and Properties of Nanochitosan. Polym. Plast. Technol. Eng. 2009, 48, 1239-1243, doi:10.1080/03602550903159069. 